\title{
Clarifying concussion in youth rugby: recognise and remove
}

\author{
L Hodgson $^{1,2}$, J Patricios $3,4,5$ \\ ${ }^{1}$ Academic Orthopaedics, University of Nottingham, UK \\ ${ }^{2}$ Corobeus Sports Consultancy Ltd, UK \\ ${ }^{3}$ Sports Concussion South Africa, Johannesburg, South Africa \\ ${ }^{4}$ The Section of Sports Medicine, Faculty of Health Sciences, University of Pretoria, Pretoria, South Africa \\ ${ }^{5}$ The Department of Emergency Medicine, Faculty of Health Sciences, University of the Witwatersrand, \\ Johannesburg, South Africa
}

Correspondence to Dr L Hodgson, Academic Orthopaedics, Trauma and Sports Medicine, Queens Medical Centre, University of Nottingham, Nottingham NG7 2UH, UK; lisa.hodgson@ live.com

\section{The issue}

In the April 2015 issue of BJSM, Kirkwood et al ${ }^{1}$ drew attention to concussion in youth rugby, concluding with four recommendations, most of which are well founded. One would agree there is a lack of medical personnel at youth games, a need for increased awareness of concussion management and a requirement for generalised data collection. However, suggesting rugby alone should 'never be compulsory' is highlighting that it might perhaps be an unsafe sport to play.

\section{Concussion awareness is positive}

Concussion is a 'hot topic' and Kirkwood et al ${ }^{1}$ highlight concussion as the most commonly reported rugby match injury. This is due to heightened concussion awareness and diagnosis with the introduction of the new pitch side assessment tools rather than an increase in true incidence. ${ }^{2}$ This is a good outcome, if we know about the injury and establishing it is now better recorded, it can be suitably managed.

\section{Identifying concussion and managing appropriately}

A review in rugby league observed 470 concussions over seven seasons $(2.65 / 1000 \mathrm{~h})$. The odds of sustaining one concussion was $7 \%$, whereas the chances of receiving two or more was $1 \%$ and three or more was $0.2 \% .^{3}$ In rugby, concussions managed correctly and fully resolved have not been shown to have detrimental effects on future concussions ${ }^{3}$ and in general there is a lack of evidence linking appropriately managed concussions in other sports to detrimental future effects. ${ }^{4}$ When a head injury is not managed well, players run a significant risk of lengthening their symptoms, developing postconcussion syndrome and, in a few tragic cases, suffer second impact syndrome (SIS). ${ }^{4}$ The risks of SIS would be minimised, perhaps negated, if the first injury was managed correctly and any suspected concussion removed from the field. The next appropriate step is well-informed medical evaluation and return-to-play guidance.

\section{Maintain perspective}

The debate should move on from 'what is a concussion' and the inferred relationship between concussion and chronic traumatic encephalopathy. Focus should be on education and recognition. 
Kirkwood et al emphasise the inherent risk of receiving a concussion while playing any sport inclusive of rugby. One must support the view that those taking care of the adolescent athlete should be well informed in concussion management and know the detrimental effects if the injury is mismanaged. Bearing in mind most rugby sessions will not have expert clinicians in attendance, coaches, referees and first aiders have a key role to play. They should be aware concussion may mimic severe traumatic brain injury and have significant sequelae if ignored. It is out of their remit to make a diagnosis. They must be cautious, remove the player immediately and seek medical attention.

\section{Spread the word rather than jeopardise the sport}

While Zurich $2012^{5}$ provides guidance to the clinical population, there is also much we do not know. ${ }^{6}$ Healthcare professionals debate how to interpret and manage concussion, how do we expect the layperson to do better? Concussion is one of the hardest sporting injuries to diagnose, especially for those inexperienced in its management.

We must propagate what we do know rather than discourage participation in sports with significant physical, social and cognitive benefits. ${ }^{7}$ How far current knowledge disseminates down to lay carers volunteering at youth sport is currently indefinable, but that the message requires dissemination is indisputable. Our role as clinicians is to keep the player participating where the benefits of doing so outweigh the risks and rugby offers significant benefits to a diverse cohort of participants.

\section{Clarify the issues...}

Concussion can have devastating outcomes if not identified or managed accordingly, something that is not rugby-specific. ${ }^{4}$ Cycling has a high incidence of head injuries ${ }^{8}$ yet there is hardly a clarion call for banning bicycles! While adolescent injuries may go on being under-reported and concussion as an evolving injury might not always be obvious, heightening awareness of the injury and its serious effects if mismanaged will aid in dealing with the issue rather than avoiding a sport loved worldwide.

Medical responsibilities should include dispelling myths such as: 'concussion only occurs when a player loses consciousness'; 'concussion symptoms are obvious'; 'helmets will protect you.' Work on getting the right messages to the right people and we are half way to managing this injury appropriately. World Rugby has a simple and poignant message not alluded to by Kirkwood and colleagues: 'Recognise and Remove' and their age-specific guidelines ${ }^{9}$ effectively elaborate on this principle. Following this significant intervention, return to play must remain an informed medical decision.

Future research will advise on the cumulative effects of subconcussive events, but for now we can focus on what we can achieve without excluding rugby. The injury is not getting worse, our knowledge of its presence is improving, now let us improve our management strategy without speculating.

\section{...and send the right message}

Parents, coaches and first aiders with a knowledge of concussion and the Zurich guidance should be available pitch side at every sporting event, especially school and club level involving children. They should never return a player to play or training with suspected 
concussion. Finally, whilst taking our medical roles seriously and avoiding risk, we should acknowledge the many benefits of participating in sports including rugby.

\section{Footnotes}

- Twitter Follow Jon Patricios at @ jonpatricios

- Competing interests None declared.

- Provenance and peer review Not commissioned; externally peer reviewed.

\section{References}

1. Kirkwood G, Parekh N, Ofori-Asenso R, et al. Concussion in youth rugby union and rugby league: a systematic review. Br J Sports Med 2015;49:506-10. doi:10.1136/bjsports-2014-093774

2. England Professional Rugby Union Injury Surveillance Project Steering Group. England Professional Rugby Injury Surveillance Project 2012-2013 Season Report.http://www/rfu/com/takingpart/ /media/files/2014/takingpart/injuryauditrepor t2014.ashx (accessed 11 Dec 2014)

3. Hodgson L, Walker $\mathrm{P}$, Gissane $\mathrm{C}$, et al. A seven year retrospective study on concussion: The time between concussions, the odds of sustaining a subsequent concussive injury and return to play timelines in professional rugby league Masters thesis. University of Nottingham, 2015.

4. Cantu R, Hyman M. Concussions and Our Kids. Boston, New York: Mariner Books, Houghton Mifflin Harcourt, 2013.

5. McCrory P, Meeuwisse WH, Aubry M, et al. Consensus statement on concussion in sport: the 4th International Conference on Concussion in Sport held in Zurich, November 2012. Br J Sports Med 2013;47:250-8. doi:10.1136/bjsports-2013-092313

6. Patricios JS, Makdissi M. The sports concussion picture: fewer 'pixels', more HD. Br J Sports Med 2014;48:71-2. doi:10.1136/bjsports-2013-093285

7. http://www.englandrugby.com/my-rugby/players/player-health/rugby-for-a-healthylifestyle

8. Ruest N, Nguyen M, Embree T, et al. Mountain bike terrain park injuries: an emerging cause of morbidity. Br J Sports Med 2011;45:312. doi:10.1136/bjsm.2011.084038.8

9. World Rugby Player Welfare, Putting Players First. IRB Concussion Management. http://www.irbplayerwelfare.com/concussion (accessed 16 Dec 2014). 